\title{
Tiroidectomía en paciente embarazada con enfermedad de Graves sin respuesta a tratamiento médico: reporte de caso
}

\section{Thyroidectomy in Pregnant Patient with Grave's Disease Without Response to Medical Treatment: Case Repor}

Recepción: 11/10/2019 | Aceptación: 21/01/2020

\author{
LeONARdo JaVier Rojas Melo \\ Médico internista endocrinólogo, Unidad de \\ Endocrinología, Hospital Universitario San Ignacio, \\ Bogotá, Colombia \\ Viviana Cruz Ramírez \\ Médica residente de Patología, Facultad de Medicina, \\ Pontificia Universidad Javeriana, Bogotá, Colombia \\ Elly Morros-GonzÁlez \\ Médica residente de Geriatría, Facultad de Medicina, \\ Pontificia Universidad Javeriana, Bogotá, Colombia \\ Angélica Imitola Madero \\ Médica internista endocrinóloga, Unidad de \\ Endocrinología, Hospital Universitario San Ignacio, \\ Bogotá, Colombia
}

a Autor de correspondencia: leonardorojasmd@yahoo.com

Cómo citar: Rojas Melo LJ, Cruz Ramírez V, MorrosGonzález E, Imitola Madero A. Tiroidectomía en paciente embarazada con enfermedad de Graves sin respuesta a tratamiento médico: reporte de caso. Univ. Med. 2020;61(3). https://doi.org/10.11144/Javeriana. umed61-3.grav

\section{RESUMEN}

Objetivo: Describir el caso de una paciente embarazada con enfermedad de Graves sin respuesta a antitiroideos, a fin de exponer las opciones terapéuticas adicionales disponibles como colestiramina, corticoesteroides, yoduro de potasio, plasmaféresis y tiroidectomía.

Materiales y métodos: Se presenta el caso de una mujer de 18 años de edad con embarazo de 25 semanas y enfermedad de Graves desde hace 2 años, tratada con altas dosis de metimazol y propranolol. Se consideró diagnóstico de preeclampsia y tormenta tiroidea probable (Burch Wartofsky 25).

Resultados: La evolución clínica posoperatoria fue satisfactoria; la paciente fue dada de alta con tratamiento sustitutivo con levotiroxina de $100 \mu \mathrm{g} /$ día. A las 39 semanas de gestación, nació por cesárea una niña sana con peso $3200 \mathrm{~g}$ con medición de TSH neonatal en 6,21 mU/ml (0-15).

Conclusiones: La tiroidectomía al final del segundo trimestre es una alternativa terapéutica favorable en pacientes embarazadas con enfermedad de Graves sin respuesta al tratamiento médico.

\section{Palabras clave}

tiroidectomía; enfermedad de Graves; hipertiroidismo; embarazo; crisis tiroidea.

\section{ABSTRACT}

Objective: To describe the case of a pregnant patient with Graves' disease who presented inadequate response to antithyroid medications with the aim to present the alternative therapeutics options such as cholestyramine, steroids, potassium iodide, plasmapheresis and thyroidectomy. 
Material and methods: 18 years-old-female patient, with 25-week pregnancy and history of Graves' disease for 2 years, treated with high doses of methimazole and propranolol.

Results: Post-surgical evolution was satisfactory; patient was discharged with levothyroxine $100 \mathrm{mg} /$ day treatment. At the 39th week of gestation, a healthy female baby was born, weighting 3200 grams with a normal neonatal TSH $6.21 \mathrm{mU} / \mathrm{ml}(0-15)$.

Conclusions: Thyroidectomy at the end of the second trimester in pregnant women with Graves' disease may be a favorable therapeutic alternative in case of inadequate response to medical treatment.

Keywords

thyroidectomy; Graves' disease; hyperthyroidism; pregnancy; thyroid crisis.

\section{Introducción}

La incidencia del hipertiroidismo en el embarazo es del 0,05\%, siendo más frecuente la etiología autoinmune, principalmente la enfermedad de Graves, en el $85 \%$ de los casos $(1,2)$. Entre las principales manifestaciones clínicas de la enfermedad se encuentran signos y síntomas de tirotoxicosis, bocio, orbitopatía y dermatopatía $(3,4)$. El hipertiroidismo afecta el desarrollo del embarazo y representa un alto riesgo para la salud materna y fetal (5). El pobre control de la tirotoxicosis en la madre se ha asociado con hipertensión inducida por embarazo, aborto, óbito, parto prematuro, falla cardiaca materna, bajo peso del recién nacido, restricción del crecimiento intrauterino e hipertiroidismo fetal (6). La primera línea de tratamiento son los medicamentos antitiroideos, los cuales son efectivos la mayoría de las veces. En caso de falla terapéutica o contraindicación para esta terapia, una de las alternativas terapéuticas es la tiroidectomía; sin embargo, es poca la experiencia descrita para esta intervención en pacientes embarazadas. En este artículo, describimos el caso de una paciente embarazada sin respuesta al tratamiento médico, en quien se consideró la realización de una tiroidectomía al final de su segundo trimestre, con desenlace favorable.

\section{Reporte de caso}

Al servicio de urgencias del Hospital Universitario San Ignacio, en Bogotá (Colombia), se presentó una mujer de 18 años de edad, de etnia mestiza, quien cursaba su segundo embarazo de 25 semanas. Consultó por un cuadro clínico de 3 días de evolución de edema en sus miembros inferiores, cefalea, palpitaciones y temblor de manos en reposo. Como antecedentes, la paciente tenía diagnóstico de hipertiroidismo por enfermedad de Graves desde hacía 2 años y estaba en tratamiento con medicamentos antitiroideos, con dificultades para la adherencia y el seguimiento. Fue diagnosticada en octubre del 2014 (tabla 1) y había iniciado tratamiento con 35 miligramos al día de metimazol. En diciembre del mismo año, la paciente quedó embarazada, por lo cual en el primer mes de la gestación se cambió el tratamiento a $350 \mathrm{mg}$ por día de propiltiouracilo.

Tabla 1.

Seguimiento de la función tiroidea

\begin{tabular}{|c|c|c|c|}
\hline Fecha & TSH $(\boldsymbol{\mu U} / \mathbf{m l})$ & $\begin{array}{c}\text { T4 libre, T4L } \\
\mathbf{n g} / \mathbf{d l ~ ( 0 , 8 - 1 , 9 )}\end{array}$ & $\begin{array}{c}\text { T3 total, T3T } \\
\mathbf{n g} / \mathbf{m l} \mathbf{( 0 , 9 1 - 2 , 1 8 )}\end{array}$ \\
\hline $4 / 11 / 2014$ & 0,00 & 5,34 & \\
\hline $11 / 12 / 2014$ & $<0,005$ & 7,77 & \\
\hline $9 / 2 / 2015$ & 0,00 & 5,18 & \\
\hline $2 / 4 / 2016$ & & $>6$ & \\
\hline $14 / 4 / 2016$ & & 7,7 & 4,06 \\
\hline $24 / 5 / 2016$ & 0,018 & 7,77 & 6,51 \\
\hline $4 / 9 / 2016$ & & 7,77 & \\
\hline $1 / 10 / 2016$ & 0,01 & 5,03 & \\
\hline
\end{tabular}

TSH hormona estimulante de la tiroides.

Nota: los rangos no son específicos para cada trimestre del embarazo.

Conocida la paciente en la institución en febrero del 2015, al descartar tormenta tiroidea, y dado que había transcurrido el primer trimestre del embarazo, se consideró reiniciar el metimazol a dosis de $45 \mathrm{mg}$ por día. La paciente tuvo el parto en julio del 2015, aun cursó con preeclampsia y requirió estancia en la unidad de cuidado intensivo. Continuó sus controles en otra institución. Reingresó al hospital universitario en abril del 2016, remitida de consulta externa por 
sospecha de tormenta tiroidea con 25 puntos en la escala Burch-Wartofsky, sin orbitopatía activa. Nuevamente, volvió al tratamiento con metimazol ambulatorio a dosis de $35 \mathrm{mg}$ VO cada $8 \mathrm{~h}$ y propanolol de $80 \mathrm{mg} \mathrm{VO}$ cada $8 \mathrm{~h}$, en vista de la pobre respuesta clínica y valores muy altos de T4 libre, lo que condicionó el aumento de esta dosis extrainstitucional.

Durante la hospitalización se ajustó la dosis de metimazol a $40 \mathrm{mg}$ cada $8 \mathrm{~h} \mathrm{y} 120 \mathrm{mg}$ de propanolol cada $8 \mathrm{~h}$. Se adicionó tratamiento con colestiramina y esteroide. Se consideró candidata a terapia con yodo radioactivo; sin embargo, en julio del 2016, previo a la administración de yodo radiactivo, se documentó una gestación de 12 semanas. Se suspendió la orden de yodo y se indicó tratamiento con metimazol $(80 \mathrm{mg}$ por día); sin embargo, la paciente modificó la dosis a $60 \mathrm{mg}$ por día y a $120 \mathrm{mg}$ de propranolol cada 6 h. No se consideró el cambio a propiltiouracilo, dado que había transcurrido el primer trimestre del embarazo.

Al ingreso, se encontró una mujer con los siguientes signos vitales:

Tensión arterial: 153/65 mm Hg

Frecuencia cardiaca: 130 latidos por minuto

Temperatura: $36^{\circ} \mathrm{C}$

Así mismo, leve retracción palpebral como orbitopatía tiroidea; actividad de la oftalmopatía CAS: $1 / 7$; bocio: grado 3 , y edema: grado I de miembros inferiores.

La proteinuria en la orina de 24 horas resultó negativa, por lo cual se descartó preeclampsia. Los estudios de la función tiroidea mostraron una hormona estimulante de la tiroides (TSH) de 0,01 mU/1; T4L de 5,03 ng/dL (0,9-1,9) y T3T de $3,98 \mathrm{ng} / \mathrm{ml}(0,79-1,49)$. Se realizaron anticuerpos antirreceptores de TSH positivos en 1,78 UI/l $(<1,75)$. Se inició manejo antihipertensivo con nifedipino de $30 \mathrm{mg}$ y alfametildopa de $250 \mathrm{mg}$ VO cada $8 \mathrm{~h}$ y se adicionaron $2 \mathrm{~g}$ de colestiramina cada $8 \mathrm{~h}$, esteroide endovenoso y yoduro de potasio (lugol).

Ante la falla frente al tratamiento con medicamentos antitiroideos, el caso de la paciente fue llevado a junta médica de tiroides, donde se consideró una tiroidectomía, que se realizó en la semana 27 de gestación. El principal hallazgo intraoperatorio fue una glándula tiroides aumentada de tamaño, hipervascularizada, de fácil sangrado. La patología evidenció parénquima tiroideo normal, intercalado con zonas donde se reconocen folículos de tamaño variable con material coloide en su interior, con congestión vascular y rodeados por gran infiltrado inflamatorio linfoplasmocitario (figura 1). La evolución clínica posoperatoria fue satisfactoria; la paciente fue dada de alta con tratamiento sustitutivo con $100 \mathrm{mg} /$ día de levotiroxina. A las 39 semanas de gestación, nació por cesárea una niña sana con $3200 \mathrm{~g}$ de peso y medición del TSH neonatal en $6,21 \mathrm{mU} /$ $\mathrm{ml}(0-15)$.

\section{Figura 1.}

Descripción de placa histológica de la tiroides.

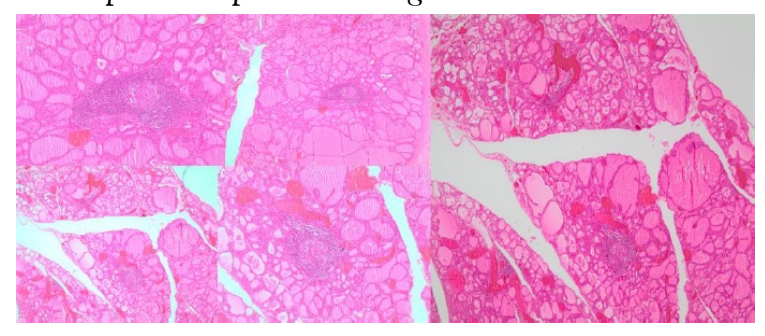

A menor aumento, los cortes muestran áreas normales del parénquima tiroideo, intercalado con zonas donde se reconocen folículos de tamaño variable con material coloide en su interior, con congestión vascular y rodeados por gran infiltrado inflamatorio linfoplasmocitario. A mayor aumento, se evidencian folículos tiroideos rodeados por gran infiltrado inflamatorio de predominio linfocitario, que configuran folículos linfoides con centros germinales. Algunas paredes foliculares se encuentran infiltradas por células inflamatorias con destrucción folicular y colapso

\section{Discusión}

El embarazo genera un gran impacto en la anatomía de la glándula tiroides y su función, por ejemplo, se produce un aumento en el tamaño de la glándula tiroides, un $10 \%$ en países con adecuada ingesta de yodo y de un 20 a un $40 \%$ en áreas con deficiencia de yodo. La producción de hormonas tiroideas T3 y T4 se incrementa en cerca del $50 \%$, así como en un $50 \%$ el requerimiento diario de yodo $(1,2)$. 
Durante el primer trimestre de la gestación los cambios hormonales modifican la función tiroidea. Así, aumenta la cantidad de gonadotropina coriónica humana, cuya estructura es similar a la subunidad $\alpha$ de la TSH, lo cual incrementa la producción de $\mathrm{T} 4$ y T3 libre y disminuye las concentraciones de TSH. Por otra parte, también aumentan los niveles de T3 y T4 totales sin que varíen los valores de la fracción libre, ya que se acrecientan los niveles de la globulina fijadora de hormonas tiroideas, debido a su síntesis por acción de los estrógenos y disminución del aclaramiento renal (7).

El hipertiroidismo en la enfermedad de Graves se da por anticuerpos tipo inmunoglobulina $G$ circulantes, que se unen y activan el receptor de tirotropina, acoplado a proteínas $G$ (3). De esta manera, el estímulo de la glándula tiroides causa hipertrofia e hiperplasia folicular, lo cual incrementa la producción de T3 y T4 entre un 20 y un $30 \%(6,7)$. El cuadro clínico del hipertiroidismo se caracteriza por pérdida de peso, hiperfagia, intolerancia al calor, dificultad para conciliar el sueño, aumento del hábito intestinal, debilidad muscular proximal, palpitaciones, temblor, hiperreflexia, piel caliente y húmeda, palpitaciones y ansiedad. En el examen físico se pueden encontrar: fiebre, taquicardia, oftalmopatía, bocio e hipertensión arterial de predominio sistólico. Hallazgos menos frecuentes son el mixedema pretibial y la acropaquia tiroidea. Cabe resaltar la importancia de la evaluación de la oftalmopatía en severidad y actividad, que requiere tratamiento adicional e independiente del tratamiento del hipertiroidismo $(3,4,5)$.

La enfermedad de Graves se determina por los hallazgos clínicos y el perfil tiroideo, que se caracteriza por TSH suprimida, niveles de T3 total y T4 libres elevados y la determinación de los anticuerpos antitiroideos, antitiroglobulina y antiperoxidasa, pero con especial mención de los anticuerpos antirreceptor de TSH (TRAb). Se ha descrito que los TRAb en la enfermedad de Graves pueden cruzar la barrera hematoplacentaria y afectar la función tiroidea del feto, por lo que la terapia con medicamentos antitiroideos debe garantizar una adecuada función tiroidea tanto materna como fetal, a fin de evitar el hipotiroidismo en este último $(8,9)$. Los niveles elevados de TRAb > $5 \mathrm{IU} / \mathrm{L}$ (3 veces el límite normal) en el segundo y tercer trimestres predicen hipertiroidismo neonatal con una sensibilidad del $100 \%$ y una especificidad del $43 \%$, por lo cual el feto requiere monitorización hasta la etapa posnatal, incluso si se logra eutiroidismo en la madre $(10,11,12)$.

El rango de referencia normal para la TSH durante cada trimestre de embarazo es variable; por esto, la ATA 2017 recomienda establecer rangos de laboratorio de referencia para cada trimestre (10).

Las guías internacionales recomiendan evaluar la función tiroidea según los rangos estipulados del laboratorio. En caso de no estar disponibles, las guías internacionales deberían usar los siguientes rangos para la TSH: primer trimestre: 0,1-2,5 mIU/1; segundo trimestre: 0,2-3,0 mIU/1, y tercer trimestre: 0,3-3,0 mIU/1 (10). Los rangos del laboratorio para T4L y T3T son 0,8-1,9 ng/dL y $0,91-2,18 \mathrm{ng} / \mathrm{mL}$, respectivamente (10).

El objetivo del tratamiento del hipertiroidismo es mantener las concentraciones de T4T/T4L en el límite superior de la normalidad. Se debe utilizar la menor dosis de medicamento antitiroideo posible; en el primer trimestre, se recomienda propiltiouracilo, y a partir del segundo trimestre, continuar el tratamiento con metimazol (11). El uso de este último en el primer trimestre se ha relacionado con anormalidades congénitas (por ejemplo, aplasia cutis, atresia de coanas, fístula traqueoesofágica, pezones hipoplásicos y retardo del neurodesarrollo). El propiltiouracilo se asocia con falla hepática en $<1 \%$ de las embarazadas. Dos metanálisis recientes demostraron una asociación con malformaciones fetales, como quistes y anormalidades de las vías urinarias y malformaciones cardiovasculares. Estos efectos se observan principalmente entre las semanas 5 a 10 de la gestación, que constituyen el periodo de mayor teratogénesis $(12,13,14)$.

$\mathrm{Si}$ la paciente tenía un diagnóstico de enfermedad de Graves y cumple criterios clínicos y bioquímicos que sugieren remisión, se puede retirar el medicamento antitiroideo y realizar 
seguimiento cercano de la función tiroidea. En general, los factores de riesgo para falla al antitiroideo o recaída en el hipertiroidismo son: haber recibido tratamiento por corto tiempo (menos de 6 meses), TSH suprimida a pesar del tratamiento, requerimiento de dosis de metimazol mayor a $5-10 \mathrm{mg} /$ día, orbitopatía o bocio grande y altos niveles de TRAb $(6,11,15)$.

Como alternativa terapéutica, para pacientes embarazadas, en Japón se han realizado estudios con tabletas de 10-30 mg de yoduro de potasio que han demostrado seguridad en los desenlaces materno-fetales. No hay otros datos de seguridad de terapia con yodo en pacientes embarazadas. Se ha considerado que, de manera crónica, el yodo puede inhibir la organificación del yodo en el feto; sin embargo, no hay evidencia de que el uso por corto tiempo sea perjudicial $(15,16,17)$. Otra opción es la colestiramina, que se une a las hormonas tiroideas, impiden la circulación enterohepática y disminuyen su concentración sérica; dado que no se absorbe, no produce lesión en el feto, pero podría interferir con la absorción de otros nutrientes y vitaminas para la madre, lo cual requiere estrecha vigilancia. La terapia de ablación con yodo radioactivo $\left(\mathrm{I}^{131}\right)$ está contraindicada durante el embarazo y la lactancia, debido a la teratogenicidad $(11,12,16)$.

La cirugía es una alternativa terapéutica cuando se presentan efectos adversos a los medicamentos antitiroideos (usualmente cutáneos, hepáticos o medulares), falla al tratamiento con antitiroideos (dosis de metimazol más de $30 \mathrm{mg} /$ día, propiltiouracilo más de $450 \mathrm{mg} /$ día) o está contraindicado el tratamiento antitiroideo (10). La cirugía se debe evitar en el primer trimestre del embarazo, ya que se relaciona con aborto y teratogénesis por agentes anestésicos, así como en el tercer trimestre, ya que se ha relacionado con parto pretérmino. Lo ideal es realizarla en el final del segundo trimestre de embarazo, aunque el riesgo de parto pretérmino puede persistir hasta en un $4,5-5,5 \%$ de los casos $(11,15,18)$.

Con posterioridad a la tiroidectomía total, las cantidades de TRAb disminuyen durante los tres meses siguientes; pero se han descrito factores que pueden conllevar una disminución más lenta de los valores, como lo son la orbitopatía y el tabaquismo (16). La preparación para la cirugía incluye betabloqueadores; sin embargo, se debe evitar su uso a largo plazo, porque está relacionado con alteración del flujo placentario, retardo del crecimiento intrauterino, bradicardia fetal, hipoglucemia neonatal y aborto $(11,14,19)$.

Algunas presentaciones de caso han descrito plasmaféresis como preparación para la tiroidectomía, con el fin de lograr una rápida disminución de la concentración plasmática de T3 y T4 (20). La monitorización durante el embarazo se debe realizar cada 2 a 4 semanas, mientras se logra el estado eutiroideo y, posteriormente, cada 4 o 6 semanas $(11,13,21)$.

\section{Conclusión}

En mujeres embarazadas con enfermedad de Graves, la realización de tiroidectomía puede estar indicada en el caso de falla o contraindicación al tratamiento con medicamentos antitiroideos. Se sugiere llevarla a cabo en el segundo trimestre del embarazo, con una preparación preoperatoria adecuada, para minimizar los riesgos derivados de la intervención quirúrgica y los asociados a anestésicos. Además, vigilar la evolución clínica del recién nacido para evaluar signos de hipertiroidismo fetal aislado. Es recomendable que las mujeres con enfermedad de Graves cuenten con asesoría preconcepcional, a efectos de evitar los riesgos materno-fetales del hipertiroidismo mal controlado en el embarazo.

Las políticas de salud pública que se deben implementar en el embarazo están reguladas en el Decreto 780 del 6 de mayo del 2016 y la Resolución 2626 del 2019, en las que se adopta el Modelo de Acción Integral Territorial (Maite). En el caso presentado, se puede resaltar el seguimiento a este modelo, en el cual se mejora en total plenitud la condición de la salud de la paciente. Así mismo, se resalta la articulación de los agentes del sistema de salud y los actores territoriales en torno a todas las acciones tanto clínicas y quirúrgicas como administrativas, para el logro de la estabilidad de la paciente y de su hija. En este caso, se requirió una cobertura total 
que llevó hasta última instancia el tratamiento en un caso de hipertiroidismo de difícil manejo.

Conflictos de interés: los autores no tenemos conflicto de interés para el presente artículo.

Fuentes de financiación: declaramos que para la elaboración de este artículo no se recibió financiación de entidades públicas o privadas.

\section{Agradecimientos}

Al Departamento de Patología del Hospital Universitario San Ignacio, por la revisión y lectura de la patología de esta paciente.

\section{Referencias}

1. Moleti M, Di Mauro M, Sturniolo G, Russo M, Vermiglio F. Hyperthyroidism in the pregnant woman: Maternal and fetal aspects. J Clin Transl Endocrinol. 2019;16:100190. https://doi.org/10.10 16/j.jcte.2019.100190

2. Nathan N, Sullivan SD. Thyroid disorders during pregnancy. Endocrinol Metab Clin North Am. 2014;43(2):573-97. https://doi.org/10. 1016/j.ecl.2014.02.008

3. Van Dijk MM, Smits IH, Fliers E, Bisschop PH. Maternal thyrotropin receptor antibody concentration and the risk of fetal and neonatal thyrotoxicosis: a systematic review. Thyroid. 2018;28(2):257-64.

4. Martínez Perea HV, Hernández Hernández M de la C. Hipertiroidismo y embarazo. Rev Cubana Endocrinol [internet]. 2012;23(3):299-305. Disponible en: ht tp://scielo.sld.cu/scielo.php?script $=$ sci _arttext\&pid=S1561-2953201200030 0016

5. Nachum Z, Rakover Y, Weiner E, Shalev E. Graves' disease in pregnancy: prospective evaluation of a selective invasive treatment protocol. Am J
Obstet Gynecol. 2003;189(1):159-65. https://doi.org/10.1067/mob.2003.321

6. Krajewski DA, Burman KD. Thyroid disorders in pregnancy. Endocrinol Metab Clin North Am. 2011;40(4):739-63. https://doi.org/10. 1016/j.ecl.2011.08.004

7. Nguyen CT, Mestman JH. Graves' hyperthyroidism in pregnancy. Curr Opin Endocrinol Diabetes Obes. 2019;26(5):232-40.

8. Cooper DS, Laurberg P. Hyperthyroidism in pregnancy. Lancet Diabetes Endocrinol. 2013;1(3):238-49.

9. Grigoriu C, Cezar C, Grigoras M, Horhoianu I, Parau C. Management of hyperthyroidism in pregnancy. J Med Life. 2008;1(4):390-6.

10. Alexander EK, Pearce EN, Brent GA, Brown RS, Chen H, Dosiou C, et al. 2017 Guidelines of the American Thyroid Association for the Diagnosis and Management of Thyroid Disease During Pregnancy and the Postpartum. Thyroid. 2017;27(3):315-89. https://do i.org/10.1089/thy.2016.0457

11. Ross DS, Burch HB, Cooper DS, Greenlee MC, Laurberg P, Maia AL, et al. 2016 American Thyroid Association Guidelines for Diagnosis and Management of Hyperthyroidism and other causes of Thyrotoxicosis. Thyroid. 2016;26(10):1-272. https://do i.org/10.1089/thy.2016.0229

12. Laurberg P, Andersen SL. Antithyroid drug use in early pregnancy and birth defects: Time windows of relative safety and high risk? Eur J Endocrinol. 2014;17(1):R13-20. https://doi.org/10. 1530/EJE-14-0135

13. Hoon Yim C. Update on the management of thyroid disease during pregnancy. Endocrinol Metab (Seoul). 
2016;31(3):386-91. https://doi.org/10. 3803/EnM.2016.31.3.386

14. Li H, Zheng J, Luo J, Zeng R, Feng N, Zhu N, et al. Congenital anomalies in children exposed to antithyroid drugs in-utero: A metaanalysis of cohort studies. PLoS One. 2015;10(5):1-11.

15. Laurberg P, Andersen SL. Endocrinology in pregnancy: pregnancy and the incidence, diagnosing and therapy of Graves' disease. Eur J Endocrinol. 2016;175(5):R219-30.

16. Yoshioka W, Miyauchi A, Ito M, Kudo T, Tamai H, Nishihara E, et al. Kinetic analyses of changes in serum TSH receptor antibody values after total thyroidectomy in patients with Graves' disease. Endocr J. 2016;63(2):179.

17. Laurberg P, Bournaud C, Karmisholt J, Orgiazzi J. Management of Graves' hyperthyroidism in pregnancy: Focus on both maternal and foetal thyroid function, and caution against surgical thyroidectomy in pregnancy. Eur J Endocrinol. 2009;160(1):1-8. https://doi.org/10.15 30/EJE-08-0663

18. Corvilain B, Hamy A, Brunaud L, Borson-Chazot F, Orgiazzi J, Bensalem Hachmi L, et al. Treatment of adult Graves' disease. Ann Endocrinol (Paris). 2018;79(6):618-35.

19. Li X, Liu G, Ma J, Zhou L. Risk of congenital anomalies associated with antithyroid treatment during pregnancy: a meta-analysis. Clinics (Sao Paulo). 2015;70(6):453-9.

20. Bilir BE, Atile NS, Kirkizlar O, Ko Y, Akpinar S, Sezer A, et al. Effectiveness of preoperative plasmapheresis in a pregnancy complicated by hyperthyroidism and anti-thyroid drug-associated angioedema. Gynecol Endocrinol. 2013;29(5):508-10.

21. Lazarus JH. Management of hyperthyroidism in pregnancy. Endocrine. 2014;45(2):190-4. https://d oi.org/10.1007/s12020-013-0086-9 